\title{
Una Company Town en la Bahía de Todos Santos por la International Company of México (1886) y la Mexican Land and Colonization Company (1889), ciudad de Ensenada [Baja California] México
}

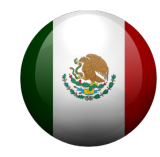

\section{Enrique Esteban Gómez Cavazos}

Arquitecto por la Universidad Autónoma de Nuevo León [Monterrey, México]. Master en Investigación en Urbanismo. Estudiante de doctorado en Urbanismo, Programa de Doctorado de la Universitat Politécnica de Catalunya [Barcelona, España]. Ensenada [Baja California] México <eecavazos@hotmail.com>.

\section{Resumen}

Las concesiones territoriales de finales del siglo XIX al capital extranjero implementan un magnífico y moderno proyecto urbanístico en Baja California, único en su tipo para la ocupación de la región del Noroeste de México. Se presenta la historia urbana de la población de Ensenada y lo que desarrollaron en ella los capitalistas norteamericanos y británicos en sus inicios. Se pretende comprobar los alcances urbanos e influencia arquitectónica que tuvo la compañía en esta población a principios del siglo XX. Se defiende la hipótesis de que su traza urbana responde más al modelo norteamericano y que contiene un rico número de edificaciones de la etapa industrial, hoy de carácter patrimonial, las cuales podrían ser consideradas un bien cultural para buscar líneas de intervención y oportunidades para su conservación.

\section{Palabras clave}

Ordenación territorial. Identidad. Patrimonio industrial.

\section{A Company Town in Bahía de Todos Santos by the International Company of Mexico (1886) and the Mexican Land and Colonization Company (1889), city of Ensenada [Baja California] Mexico}

\begin{abstract}
This article focuses on how a territorial concession of the late nineteenth century to foreign capital implements a magnificent and modern urban project in Baja California, unique in its type for the occupation of the Northwest region of Mexico. The text presents the urban history of the population of Ensenada and how the North American and British capitalists developed the city. We try to verify the urban reaches and architectonic influence that the company's had in this city at the beginning of the twentieth century, defending the hypothesis that the urban layout responds more to the North American model and that contains a rich number of constructions of the industrial era. The buildings that contain the urban grid could be considered a cultural good to find lines of intervention and heritage opportunities for conservation.
\end{abstract}

\section{Keywords}

Territorial organization. Identity. Industrial heritage. 


\section{Introducción}

La influencia del capital extranjero en el Noroeste de México a través de concesiones territoriales llevó a esta región desde su industrialización a finales del siglo XIX y principios del siglo XX a conectarse a través de puertos y vías férreas con Estados Unidos. Esto hizo que se desarrollaran ciudades diseñadas por compañías mineras, agroindustriales, urbanizadoras y ferrocarrileras en ésta región fronteriza. Hoy en día los trazados originales y su arquitectura pionera están en deterioro y no se tienen las herramientas de reflexión necesarias para su valorización histórica, ya que en la mayoría de los casos se ignora su origen urbano y por su pronta modernidad no se reconoce su patrimonio edificado. Dentro del programa colonizador que para México concibió el régimen de Porfirio Díaz, Baja California ocupó un lugar significativo en razón de lo escasamente poblado de su territorio.

La presente investigación pretende buscar en la historia urbana a partir del estudio de la industrialización en el Noroeste de México, específicamente en la península de Baja California y la estrecha relación que existió con la construcción de company towns ${ }^{1}$ y establecimientos fabriles. La definición más general de company town es la de una población diseñada por una compañía en la que gran parte o todos los bienes inmuebles, edificios, servicios públicos, hospitales, pequeños negocios, tiendas y otras necesidades o lujos dentro de sus fronteras son propiedad de una sola empresa. Este término se ha utilizado en los Estados Unidos para referirse a un pueblo o ciudad donde se espera que la lealtad a la empresa sea parte responsable de su éxito ya que la empresa es la única empleadora de la zona.

El ejemplo de la ciudad de Ensenada es un caso especial, ya que la compañía internacional de México de capital norteamericano que después pasara a capital inglés se dedicó a lotificar, vender terrenos y urbanizar como principal actividad económica. La cercanía de aproximadamente $100 \mathrm{~km}$ con Estados Unidos fue un factor importante para su desarrollo. Pudiera haber confusión con el termino company town utilizado aquí para la ciudad de Ensenada, ya que generalmente este término es empleado para poblaciones con vocación minera o agroindustrial, y aquí se emplea el termino por ser una compañía la que inicia su urbanización a través de especulación territorial extranjera.

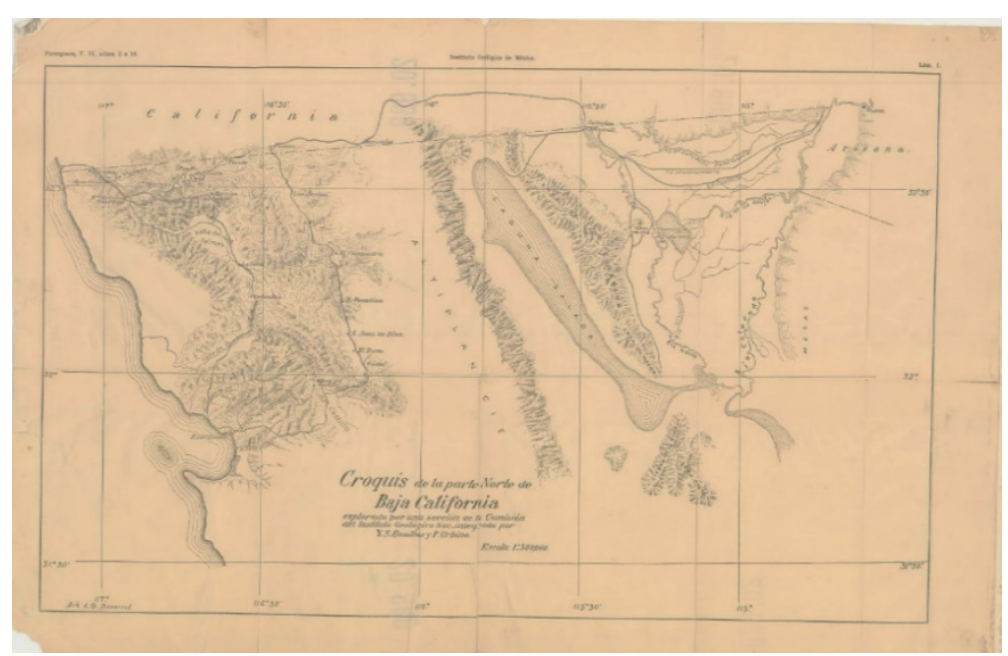

Figura 1. Mapa que muestra en la parte superior la frontera entre México y Estados Unidos en los inicios de la península de Baja California. En la parte inferior se localiza la bahía de Todos Santos donde se establece la ciudad de Ensenada en la costa del Pacífico mexicano. Fuente: Mapoteca Orozco y Berra.

Estos proyectos planeados por el capital extranjero ayudaron a construir el territorio peninsular ya que antes de la industrialización contaba con poca ocupación. Así, la modernidad se desarrolló en ciudades de nueva fundación relacionadas principalmente con actividades económicas de producción para exportación.

Acotando el estudio a la península de Baja California nos preguntamos:

- ¿Contará este territorio con una identidad urbana diferente al resto del país? ¿Puede la arquitectura industrial y los trazados por compañías ser relevantes hoy para transformar la ciudad?

\footnotetext{
${ }^{1}$ Ciudad creada por una compañía. En el caso de la ciudad de Ensenada por la International Company of México y después por la Mexican Land and Colonization Company.
} 
En respuesta, este texto se enfoca en el caso de estudio de la ciudad de Ensenada como muestra de la industrialización en Baja California. Argumentando una identidad diferente en los trazados originales y las edificaciones de esta parte del país con respecto a otras regiones y su puesta en valor como patrimonio urbano y arquitectónico. Este artículo forma parte de una investigación en curso titulada "Las ciudades de las compañías como modelo de urbanización en la península de Baja California" la cual abarca un determinado número de ciudades de nueva fundación diseñadas por capital extranjero.

\section{La influencia del ordenamiento territorial del vecino del Norte}

En Estados Unidos la ordenanza de 1785, requería dividir el territorio y vender en cuadros de 6 millas de lado que a la vez se seleccionaban en 36 partes cuadradas de 1 milla por lado (Figuras 2 y 3). Lo anterior permitía, por una parte, la fácil localización de los terrenos en el extenso territorio por ocupar y por otra, evitaba las disputas por límites. La adopción de tal división vino a reforzar la preferencia en el trazado urbano por la estructura cuadriculada (Morris, 1994).

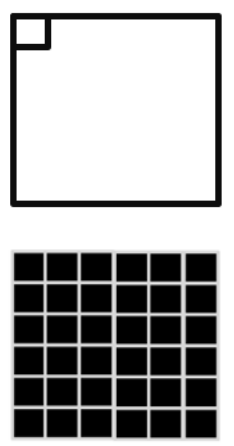

Figura 2. Esquema. La división del territorio se realizó vendiendo cuadros de 6 millas de lado que a la vez se seleccionaban en 36 partes cuadradas de 1 milla por lado. Organización territorial dictado por la Land Ordinance de 1785 de Estados Unidos.

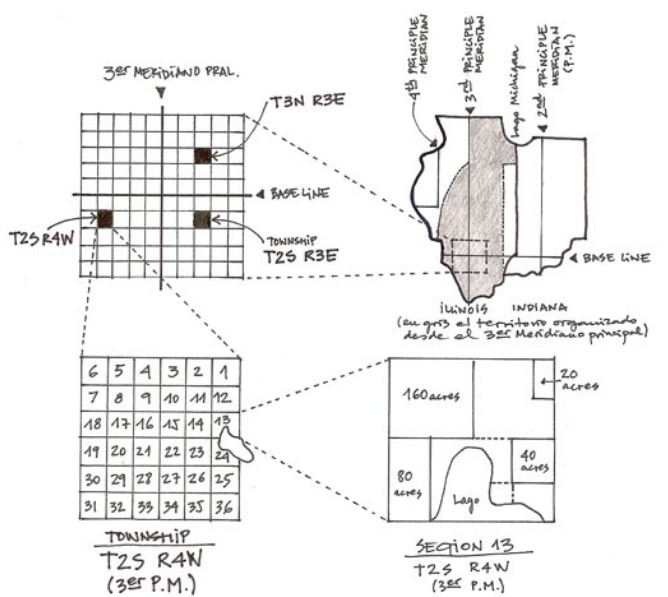

Figura 3. Esquema de organización territorial dictado por la Land Ordinance de 1785; desde los estados a los condados y de éstos a los municipios (township) y a los lotes parcelas de Estados Unidos. Fuente: Urban Networks.

Este sistema se empezó a usar en Estados Unidos de América desde la época colonial. Su gran aceptación se basa en una estructura única de fácil entendimiento y aplicación en el terreno; de apariencia racional y con la posibilidad de ofrecer a los colonos igualdad en la ubicación de sus hogares y comercios (Reps, J. W., 1979).

Así, las ciudades tuvieron formas cuadriculadas en su traza con algunas diferencias por regiones en cuanto a la concepción del uso de suelo, relacionadas con la localización y jerarquización de edificios, espacios públicos y viviendas. Indudablemente, tuvieron influencia las ideas que sobre urbanismo prevalecían en Europa (Alarcon, 2000).

A la forma cuadriculada de las ciudades en Estados Unidos también contribuyó la política del gobierno por recabar fondos de la venta de tierras al oeste de los asentamientos iniciales. Cuando el país se lanzó a la conquista del Oeste tuvo la obligación de crear numerosas ciudades a lo largo de un extenso territorio: Chicago, San Francisco, Houston, San Diego... son solo algunos ejemplos surgidos en este extraordinario esfuerzo de colonización. Todas presentan una base común: la cuadrícula en sus trazados. Estas cuadrículas urbanas formaban parte de una estrategia colonizadora más general, ya que los Estados Unidos comenzaron disponiendo de la retícula para determinar municipios, condados o estados. De forma que las ciudades eran la concreción construida en determinados puntos seleccionados en el territorio (Figura 4). Esta gran retícula cubrió la región y traspasó la frontera hacia México (Figura 5). Así, lo que los ingenieros de la International Company of México hicieron en la parte norte de la península de Baja California en México fue extender la gran red con referencia a meridianos y paralelos terrestres como lo habían hecho en California (Padilla, 1998). 


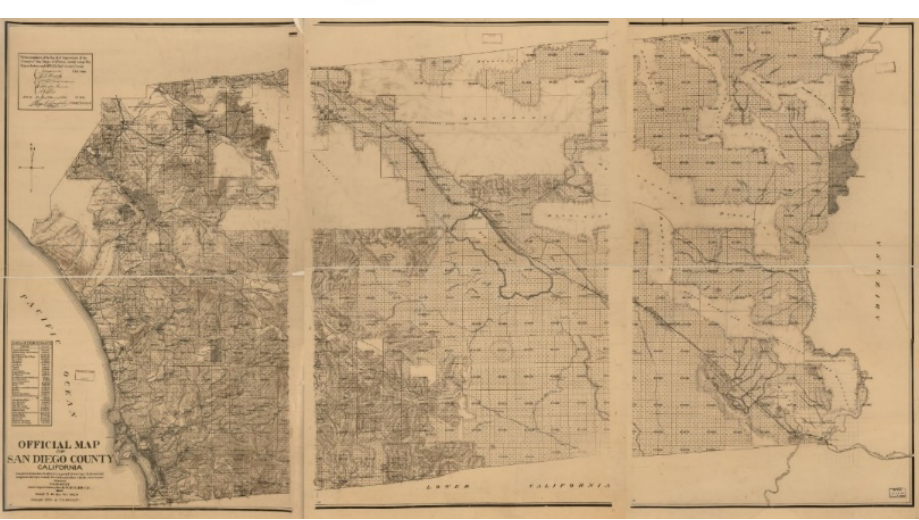

Figura 4. Plano donde se aprecia en la parte inferior la línea fronteriza entre los dos países. Fuente: Librery of the Congress of the United States, plano del condado de San Diego California 1870.

Figura 5. Mapa. Concesión territorial de $62.000 \mathrm{~km} 2$ que el gobierno mexicano otorgó a la International Company of México en Baja California. Se aprecia la cuadrícula de tipo norteamericano de organización del territorio que traspaso la frontera.

Fuente: Mapoteca Orozco y Berra, Ciudad de México.

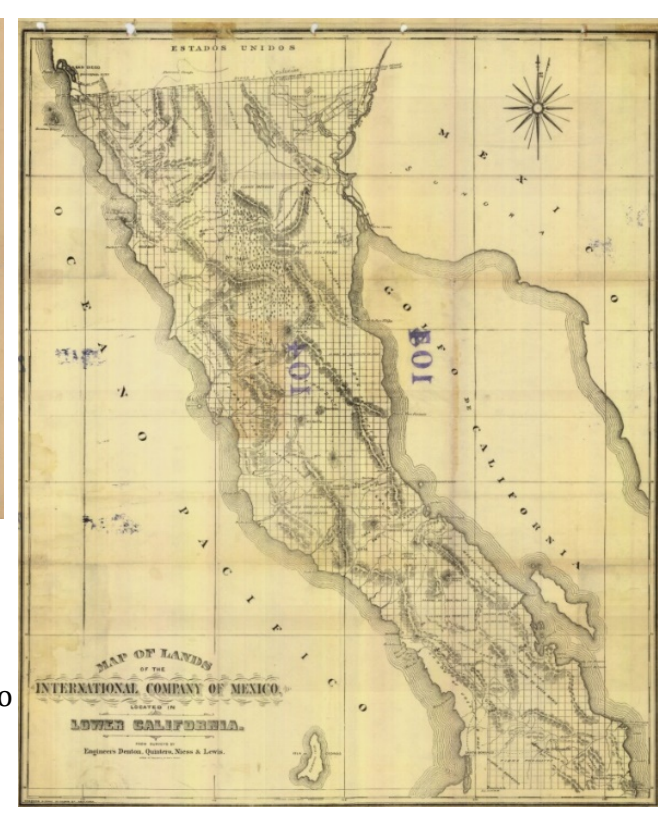

Podemos decir entonces que la región de Baja California experimentó un ordenamiento territorial diferente al resto de México de clara influencia norteamericana y que prevaleció el trazado cuadriculado. Aquí se desarrollaron las ideas urbanísticas que trajeron los norteamericanos a finales del siglo XIX, con un importante interés económico derivado del espíritu individualista de sus pobladores, que tolerarían solo el mínimo de interferencia con sus derechos de hacer lo que quisieran con su propiedad. Esta particular forma de pensar para entonces, como resultado de una motivación económica de libre mercado, es un elemento que tiene una influencia fundamental en el desarrollo de las ciudades estadounidenses.

El mecanismo de concesiones al capital extranjero en la península de Baja California fue una estrategia muy arriesgada ya que esta penetración financiera pudo haber acabado con una perdida territorial. Lo interesante aquí es la importancia que para México tenía el poblar a como diera lugar este lejano lugar, inclusive a manos de capitalistas norteamericanos, ya que fueron las concesiones territoriales las que sentaron las bases y la estructura de lo que hoy conocemos, como lo es la ciudad de Ensenada en la bahía de Todos Santos.

\section{El capitalismo especulador extranjero como urbanizador de Ensenada}

Con la expansión de Estados Unidos hacia el oeste, los especuladores en bienes raíces, planificado-res e inversionistas, pronto tuvieron la oportunidad de aplicar su experiencia como constructores de ciudades. El boom de las tierras en el sur de California surgió en 1876, cuando el Ferrocarril Southern Pacific llegó a Los Ángeles desde el norte. La población quedó ligada a la vía transcontinental que comunicaba con San Francisco. Un buen número de ciudades surgieron en esta época como aventuras especulativas en la promoción urbana (Reps, J. W., 1979).

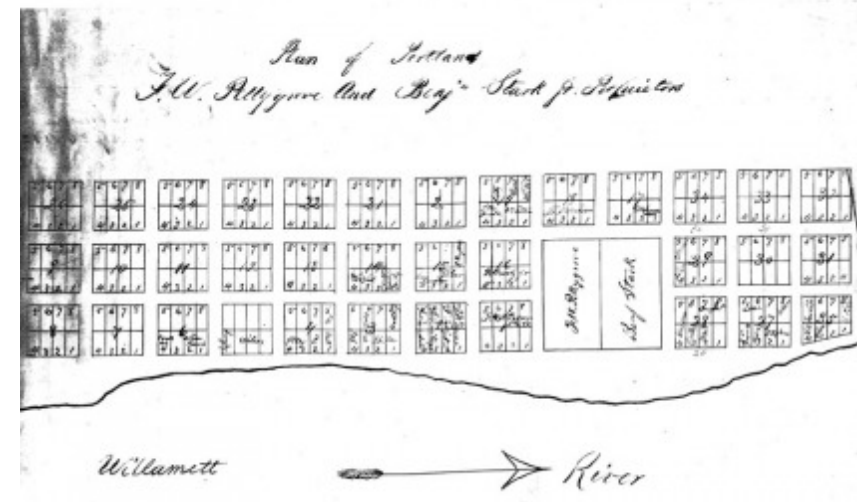

Figura 6. Un antecedente podría ser el plano de 1845 de la ciudad de Portland ubicada en el Pacifico norte de Estados Unidos. No sabemos qué relación exista entre el plan de Ensenada de 1882 en la frontera sur de California y Portland en la frontera norte de California, pero se puede observar una lotificación muy parecida inclusive la manzana está dividida en los mismos 8 lotes. Podemos pensar que en esa época la fiebre colonizadora de los Estados Unidos se expandió por la costa del Pacifico y llegaron las ideas de planificación a territorios de Baja California. Esto es solamente una hipótesis que se estudiara más afondo. 
Referente a la región de nuestro interés, la economía californiana traspasó la frontera hacia México e impactó a Baja California. Los inversionistas en bienes raíces de Estados Unidos fijaron su mirada en tierras mexicanas. Las oportunidades legales que ofrecía el gobierno federal mexicano y la demanda mundial de materias primas, hacían de la desolada península una oportunidad difícil de ignorar (Padilla, A. 1998).
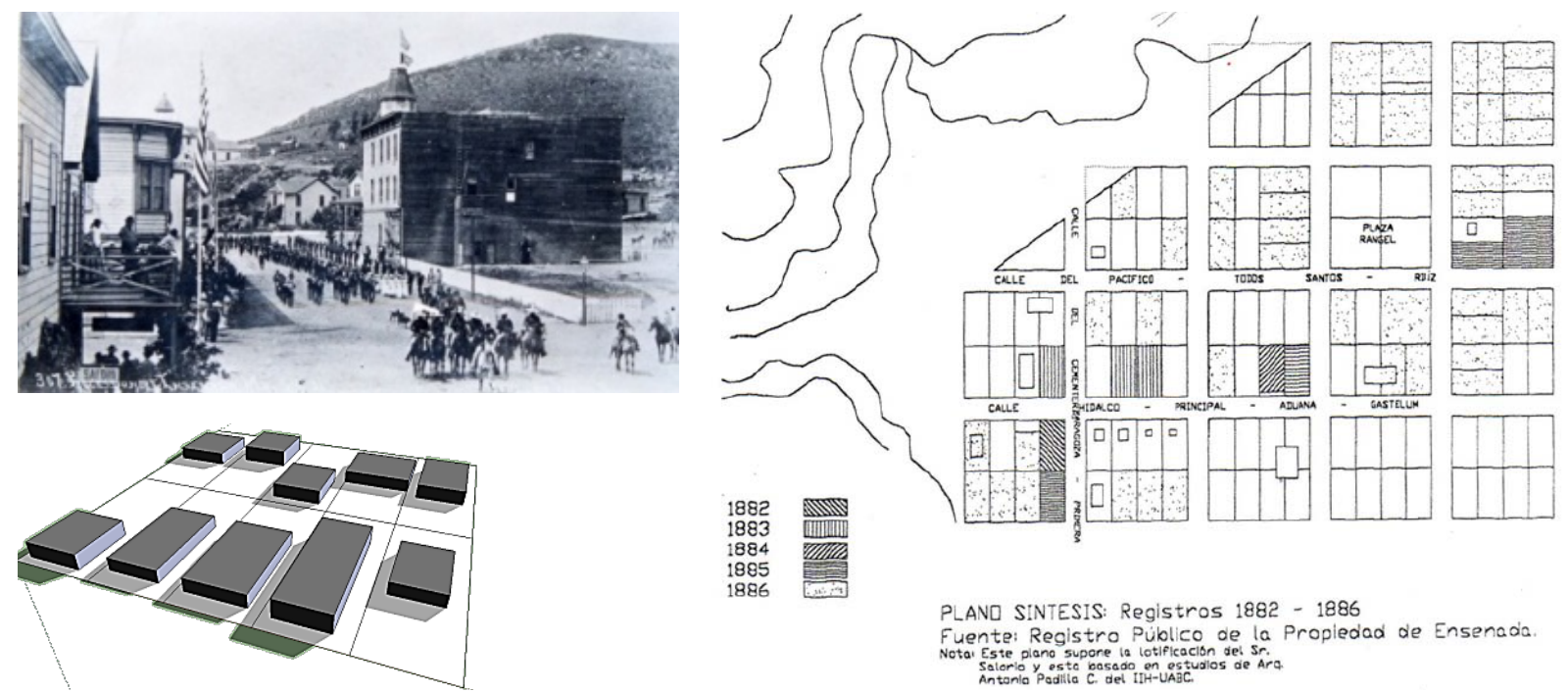

PLAND SINTESIS: Registros 1882 - 1886

Fuente: Registro Publico de la Propledad de Ensenada

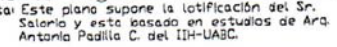

Figura 7. Fotografía de Ensenada a finales del siglo XIX y lotificación en 3D de la ocupación de las primeras manzanas. Plano más antiguo del primer cuadro de la ciudad de Ensenada donde se aprecia las primeras lotificaciones entre 1882 y 1886. Manzanas de 100m x 100m divididas en 8 lotes de 25m x 50m. Fuente: Inicios Urbanos del Norte de Baja California, Influencias e ideas, 1821-1906.

Aquí arriba en este plano se muestra la enorme labor del arquitecto e historiador Antonio Padilla Corona en la reconstrucción y redibujo a través de los datos recopilados en el Registro Público de la Propiedad de la ciudad de Ensenada que se acerca a las descripciones de Teófilo Masac de 1882-1886, antes de que la International Company of México interviniera.

Así pues, aparece en acción la International Company of México compañía de capital norteamericano que después pasaría a capital británico que aprovechó las ventajas ya mencionadas para ocupar el territorio. Esta compañía proyectó varias ciudades en la costa del pacífico, con nombres como Coronita, al norte de la bahía de Todos Santos; Ensenada, Punta Banda y San Carlos bordeando la bahía, integrándose estas 3 últimas en la llamada Colonia Carlos Pacheco; y más al sur, en San Quintín, Colnett, Willow Creek y Camalú, sin faltar planes para levantar otros poblados tierra adentro ya que contaba con mucho territorio para futuros desarrollos.

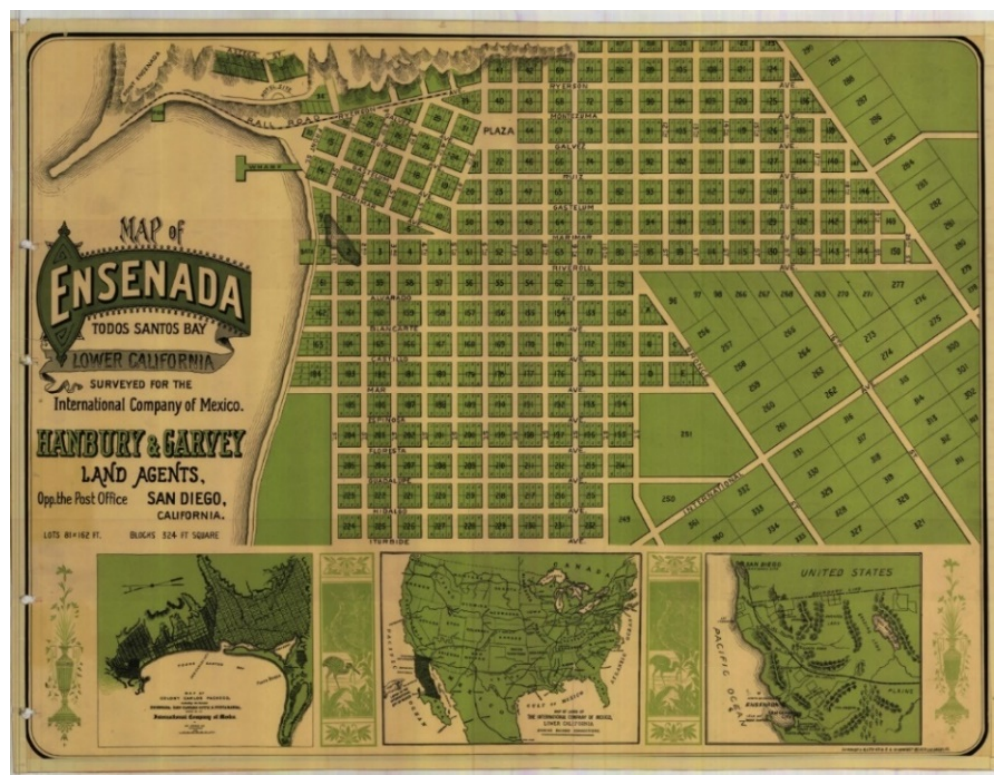

Figura 8. Plano de las lotificaciones realizado por la International Company of México en 1887 donde se aprecia el ensanche que se proyectó de la ciudad. Se puede identificar la primera retícula inclinada con respecto a la traza que siguió después. Aparece la plaza, pero no como centro y sin ningún protagonismo de edificios circundantes ya que la iglesia no se colocó alrededor de ella. Las Manzanas de $100 \mathrm{~m} \times 100 \mathrm{~m}$ fueron divididas en 8 lotes de $25 \mathrm{~m} \mathrm{x}$ $50 \mathrm{~m}$. Fuente: Mapoteca Orozco y Berra, Ciudad de México. 
Esa bonanza de bienes raíces se centró fundamentalmente en Los Ángeles y San Diego y posteriormente se extendería hacia el sur de la línea divisoria, toda vez que la compañía implementó un proyecto de desarrollo urbano en la bahía de Todos Santos en Ensenada con el propósito de atraer compradores norteamericanos. Para tal efecto se hizo una traza siguiendo algunos modelos de urbanización vigentes entonces en la Unión Americana (Piñera, 1995).

La retícula urbana más antigua del puerto de Ensenada probablemente sirvió para ordenar los caseríos de madera que se habían instalado a causa de la fiebre del oro de California. Las primeras calles y manzanas se trazaron a un costado del cerro y de la bahía, esto indica que se buscó proteger a la ciudad de los vientos dominantes.

En este párrafo lo explica de una mejor manera el arquitecto Antonio Padilla (1998):

Al protegerse del viento detrás del cerro, implicó situarse también entre otra barrera natural hacia el sur-oriente, conformada por el arroyo Ensenada. Con esta circunstancia, el planificador urbano se tuvo que ajustar al terreno disponible y deformar el contorno de su retícula, ya que la idea era mantener a toda costa la orientación original noreste-suroeste. Desde el punto de vista práctico, de haber girado la traza 45 grados se pudo haber evitado la mutilación del cuadro urbano y hubiera quedado la traza original, desapercibida dentro de la traza que desarrolló posteriormente la compañía estadounidense. Al no hacerlo, perdió en economía el trazo, pero ganó en identidad, una vez que, actualmente, se observa inconfundible de la gran traza original. (Padilla, 1998).

El proyecto de la International Company of México era un proyecto urbanístico de gran magnitud, una ciudad completa abarcando todo el terreno llano de la bahía de Todos Santos. Esta pretendía consolidar la zona junto con el estero de Punta Banda, una gran zona urbana. En la actualidad, el crecimiento ha sido lento y no como proyectó hace más de 130 años esa vieja compañía que probablemente pensaba que el desarrollo sería mucho más acelerado.

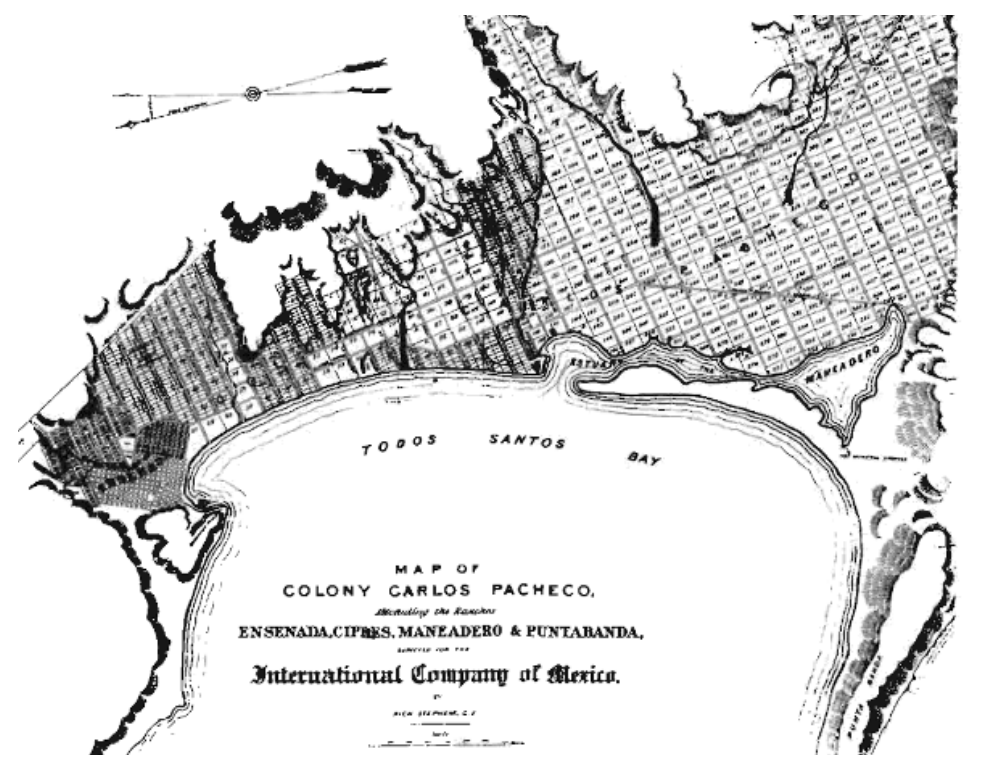

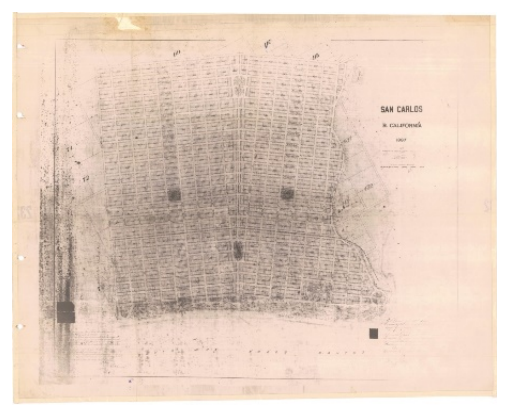

Figura 9. Plano de San Carlos 1887 y mapa de la Colonia Carlos Pacheco. Incluye los ranchos de Ensenada, Ciprés, Maneadero y Punta Banda, realizados por la International Company of Mexico, Rich Stephens. Fuente: American and English Influence on Early Development of Ensenada Baja California, de David Piñera Ramírez.

Hasta aproximadamente la mitad del siglo XIX podemos observar un lento pero continuo incremento del tamaño del módulo, que podemos atribuir a la mayor confianza en la capacidad de urbanizar, al crecimiento poblacional, y por ello, de la importancia de la ciudad. Pero asimismo encontramos proyectos tempranos que acometen una empresa de notable envergadura (Sabaté, 2014).

\section{La otra colonización: la arquitectura importada en el puerto}

No solo los capitalistas extranjeros traían las ideas urbanísticas y trazaban ciudades en territorio mexicano sino que también traían la arquitectura. Una arquitectura basada en estructuras de 
madera prefabricadas que trasportaban por barco desde Estados Unidos ya que aunque las vías ferroviarias estuvieron proyectadas por la misma compañía al puerto de Ensenada nunca llego el ferrocarril ${ }^{2}$ (el gobierno de México temía en aquella época una posible pérdida del territorio peninsular ya que las intenciones anexionistas de Estados Unidos siempre estuvieron presentes y la no construcción del ferrocarril evitó esa posibilidad al cancelar la concesión).
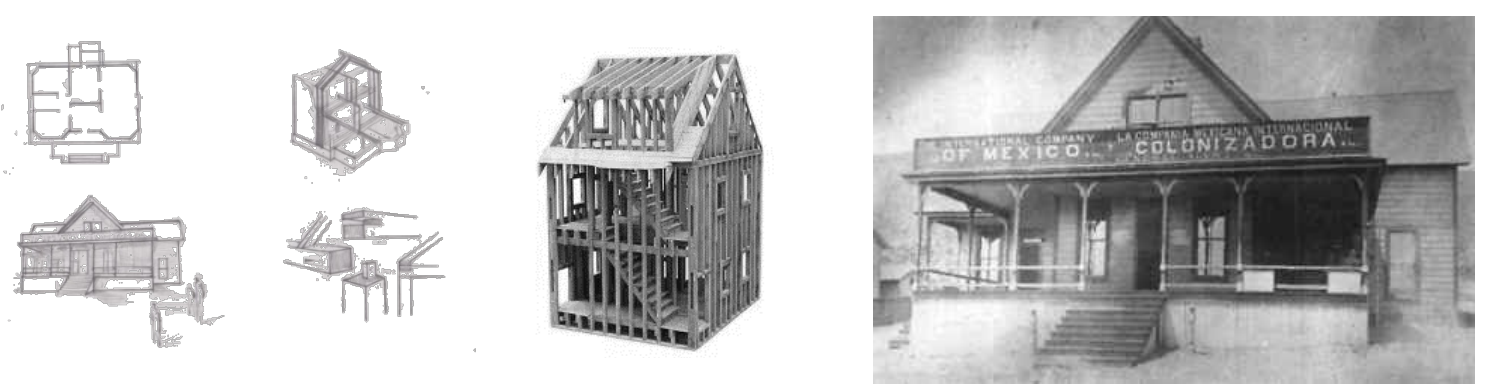

Figura 10. Esquemas del sistema constructivo Balloon Frame y edificio de la International Company of México.

Las construcciones de madera eran traídas en partes por barco desde San Diego y San Francisco California para ser ensambladas en territorio mexicano. Las primeras tiendas de artículos mineros, casas, negocios y escuelas se asentaron y conformaron un pequeño núcleo hasta que se expandió el trazado de estilo americano con calles amplias y manzanas regulares ya entrado el siglo XX.

En las investigaciones de Claudia M. Calderón se menciona lo siguiente:

Se edifica una vivienda actualmente reconocida como "tradicional", construida en madera mediante la tecnología conocida como balloon frame ${ }^{3}$, de influencia anglosajona, que se adquiría a partir del sistema de ventas por catálogos estadounidenses y se ensamblaba en Ensenada. De esta manera se contribuyó a dar una "sensación anglosajona" a la ciudad. Hoy en día hay una cantidad significativa y representativa de estas viviendas, las cuales continúan cumpliendo su objetivo de cobijo, además de representar un patrimonio cultural (Calderon \& Geffroy, 2001).
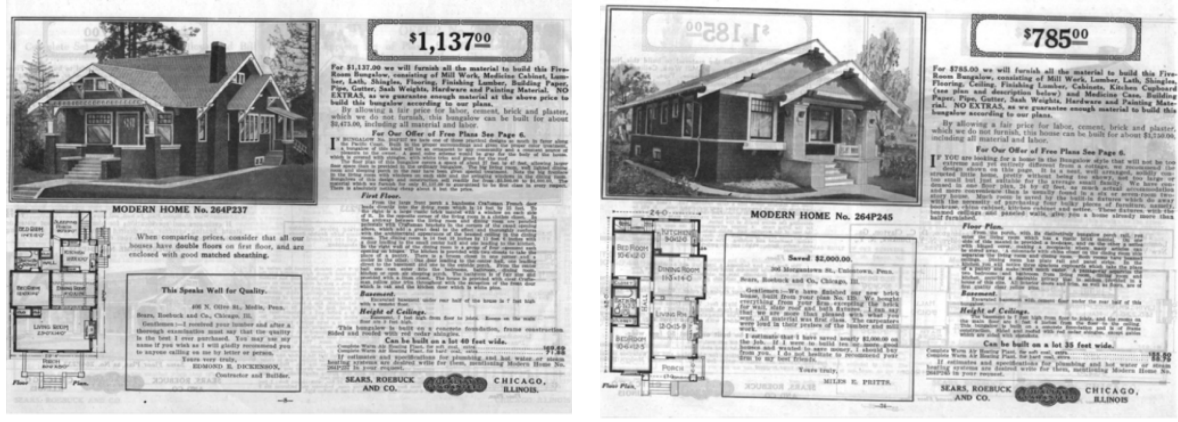

Figura 11. Catálogos norteamericanos de las edificaciones ensambladas en Ensenada provenientes de San Diego California. Fuente: Tesis doctoral "Vivienda tradicional en la ciudad y puerto de Ensenada 1882-1930" de Marcela Calderón, 2007.

Todo este impulso urbano se complementó con la construcción de algunas instalaciones clave, como las oficinas de la compañía, varias residencias, negocios y un hotel. En ellas, como es explicable, se utilizaron las formas arquitectónicas victorianas de la época. Hasta la fecha persiste el edificio de las oficinas de la empresa, convertidas hoy en sede de la delegación en Ensenada del Instituto Nacional de Antropología e Historia.

Estas referencias a continuación fueron tomas del artículo "Expresiones arquitectónicas compartidas en la frontera de Baja California" de David Piñera y Alma Bejarano.

\footnotetext{
${ }^{2}$ En el artículo de Hilarie J. Heath 2011. "El mal logrado proyecto del ferrocarril peninsular del Distrito Norte de la Baja California 1887-1892", se menciona el interés de Estados Unidos en la península y de la cancelación de la concesión ferroviaria por incumplimiento a la compañía extranjera.

${ }^{3}$ Sistema constructivo anglosajón a base de estructuras prefabricadas de madera. Con este sistema se colonizó el Oeste norteamericano.
} 

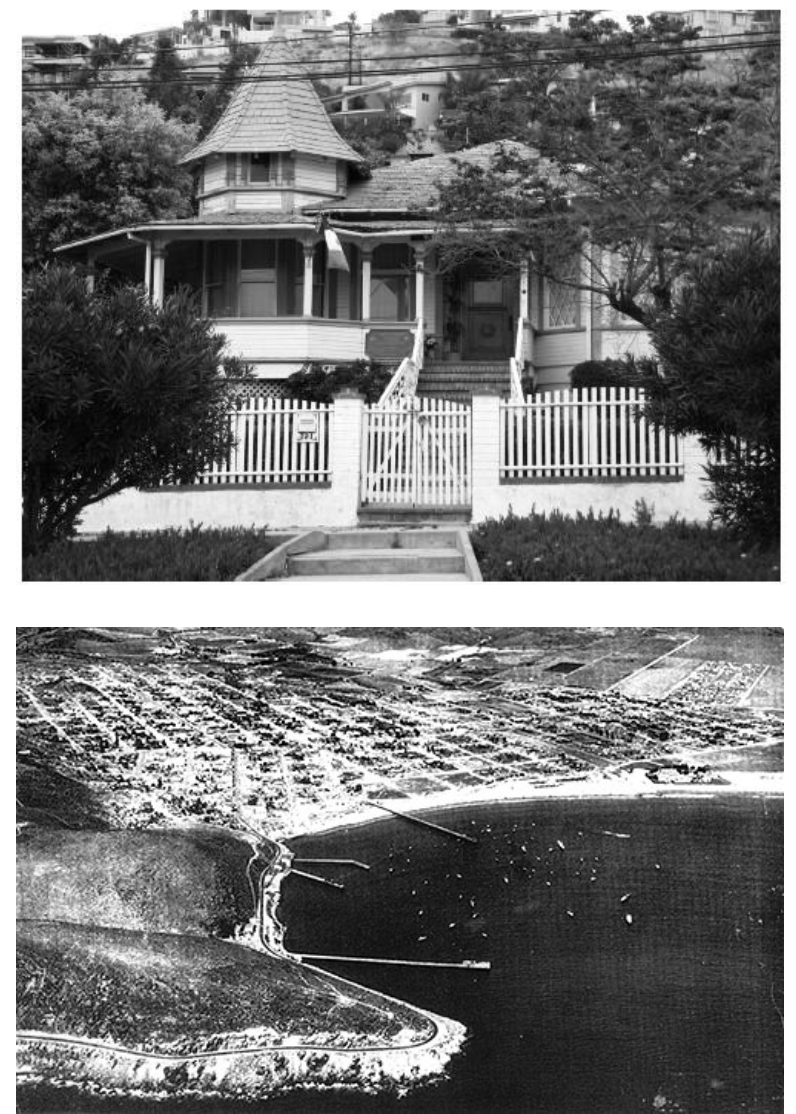

Figura 12. Residencia estilo victoriano construida en Ensenada en 1893 por Francisco Andonaegui y que ocupan actualmente sus descendientes. La residencia forma parte de los catálogos que publicaban las compañías constructoras del este de los Estados Unidos. Para construir la casa de Andonaegui las piezas de madera llegaron por barco, después de bajar hasta el Cabo de Hornos y subir después por el Océano Pacífico hasta el puerto de Ensenada (una travesía increíble que muestra la importancia de esa época para poblar esta región). Foto de Javier Hernández. Fuente: Expresiones arquitectónicas compartidas en la frontera de Baja California de David Piñera y Alma Bejarano

Figura 13. Fotografía de Ensenada ya entrado el siglo XX. Así la describe Fernando Jordán en su libro "El Otro México": Ensenada es la única cuidad que en toda la costa del Pacífico de la Baja California que no tuvo miedo al mar. A lo largo de 2.000 kilómetros de litoral (desde la frontera hasta San José del Cabo), es este el único punto donde el hombre peninsular comprendió que el océano sirve para algo más que para navegar. Aquí se desembarazó del arraigo a la tierra, heredado de los antepasados indígenas que preferían vivir tierra adentro y fundó una ciudad que baja corriendo de la montaña para venirse a bañar a la playa. Fuente: Jordán, 1956.

En la revista californiana Land of Sunshine de 1898 se le describió a Ensenada como "una agradable mezcla de sabor antiguo mexicano y confort inglés". El britanico J. R. Southworth expresó en 1899: "Las casas, fábricas y demás edificios ostentan los diversos órdenes arquitectónicos más pintorescos que resultan de una combinación del estilo antiguo de construcción con el americano moderno". También señala: "Llaman la atención las largas y anchas avenidas, las cómodas habitaciones para las familias, el cuartel general, los hoteles bien dirigidos, las espaciosas casas de comercio, y la apariencia general que da a conocer a la población como un centro de actividad comercial". Hace notar que la ciudad está "perfectamente iluminada con luz eléctrica y posee todas las mejoras modernas de las principales ciudades norteamericanas". El abogado, viajero y escritor californiano Arthur W. North visitó Ensenada en 1905, y al transmitir sus impresiones expresó: "[...] es una población relativamente nueva, americana, mexicana-inglesa, con mil quinientos habitantes, un clima delicioso y una bella ubicación sobre la curva y blanca playa de la Bahía de Todos Santos".

Estas descripciones no dejan de señalar que en los inicios de la ciudad de Ensenada parece más una pequeña ciudad norteamericana que una población mexicana, esto es un reflejo claro de la penetración financiera en la frontera entre México y Estados Unidos.

En 1917, la concesión a la compañía Mexican Land and Colonization Company —en ese momento inglesa - fue cancelada por incumplimiento de convenios, lo cual cambió el rumbo que llevaba la población, que paulatinamente se fue vinculando al desarrollo general del país. Actualmente, la ciudad ha crecido y es necesario preservar y revalorizar las edificaciones antiguas. El puerto industrial y los primeros edificios de bodegas, fábricas y casas que se encuentran en la retícula urbana que diseñó la compañía son muy buenos ejemplos para mostrar las construcciones que surgieron en la etapa industrial en el Noroeste de México.

\section{La retícula de la compañía y su oportunidad como paisaje cultural}

El Paisaje Cultural es definido como el resultado de la interacción en el tiempo de las personas y el medio natural, cuya expresión es un territorio percibido y valorado por sus cualidades culturales, producto de un proceso y soporte de la identidad de una comunidad. Como hemos visto, la parte 
central de la ciudad de Ensenada en Baja California cuenta con una identidad urbana diferente al resto de México y su población es una mezcla de todas las entidades del país.

Estudios recientes como "Patrimonio Cultural en Ensenada México. Itinerario y Preservación" por la arquitecta Claudia M. Calderón y los arquitectos Cuauhtémoc Robles y Carlos E. Aguilar se han tomado de referencia y ayudan a detectar 11 edificaciones en la traza más antigua. De las 85 de un área mayor que enlista el Instituto Nacional de Antropología e Historia (INAH) ellos hacen una magnífica labor de reconocimiento patrimonial en su guía- itinerario de la zona centro de la ciudad. También hablan de la pérdida del patrimonio cultural en la primera traza urbana, que se ha estimado en casi un $50 \%$, haciendo énfasis en conservar lo que aún pervive. Esto permite al individuo identificarse con el lugar ${ }^{4}$. De aquí la importancia de conservar los bienes culturales.
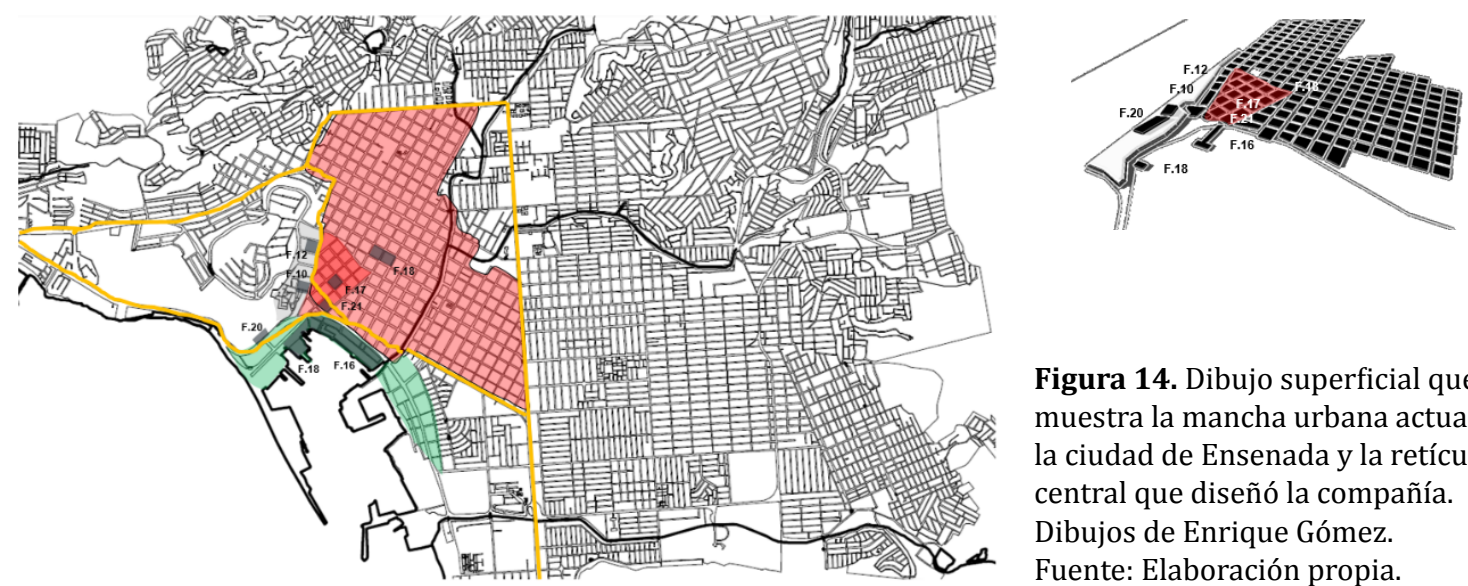

Figura 14. Dibujo superficial que muestra la mancha urbana actual de la ciudad de Ensenada y la retícula central que diseñó la compañía. Dibujos de Enrique Gómez. Fuente: Elaboración propia.

La finalidad de este artículo es complementar los anteriores estudios y presentar de forma general la manera en que se ordena este territorio, mencionando los factores más importantes que construyeron su historia urbana. Nos planteamos finalmente un reconocimiento de ese patrimonio industrial desapercibido, esas construcciones de finales del siglo XIX y principios del siglo XX en el puerto de Ensenada que no muchos conocen como objeto de conservación de la arquitectura del periodo industrial. Algunos ejemplos ya no existen pero podrían ser reconocidos por medio de fotografías en el sitio para transmitir su historia como por ejemplo los primeros muelles y otros edificios.

Figura 15. Esquemas de las manzanas de $100 \mathrm{~m} x$ $100 \mathrm{~m}$ y lotificaciones que muestran la ocupación actual, se puede apreciar la evolución y como la mayoría de la parcelación antigua que realizó la compañía sigue dividiendo los lotes actuales con variaciones evidentes después de casi 135 años de que fueron trazados. Fuente: Elaboración propia.
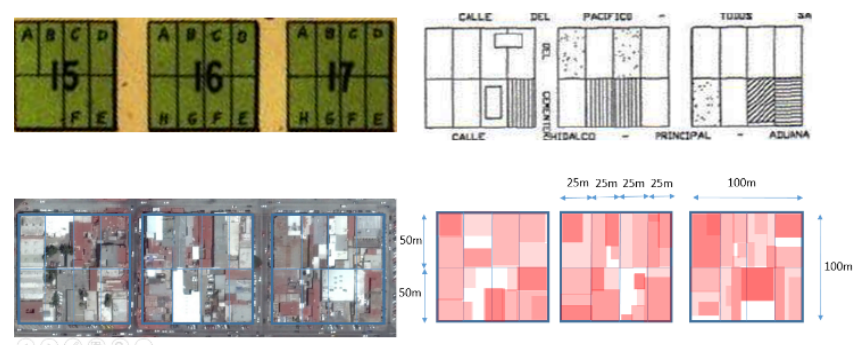

La UNESCO también define al patrimonio industrial como aquella parte del patrimonio cultural vinculada a las actividades realizadas con el fin de satisfacer necesidades humanas. Incluye bienes muebles e inmuebles, tales como: maquinas, artefactos, herramientas, chimeneas, talleres, bodegas, generados por las actividades productivas del hombre a partir de la Revolución Industrial.

Conscientes de que se necesita mucho para proteger herencia tan valiosa, de los cambiantes procesos económicos, sociales y legales, instituciones como ICOMOS ${ }^{5}$ y TICCIH $^{6}$, han promovido el

\footnotetext{
${ }^{4}$ Al revisar ese primer trazo de la ciudad de Ensenada donde viví mi infancia en la década de los 80’s entre la calle Ryerson y la calle Tercera uno se reconoce a sí mismo en cada manzana y el sentido de pertenecía aparece entre las calles, casas de madera y edificios que aunque surgieron en la época industrial son patrimoniales ya que representan nuestra identidad, que aunque reciente, no es menos valiosa que otras.

${ }^{5}$ Consejo Internacional de Monumentos y Sitios. Es una asociación civil no gubernamental ubicada en París, ligada a la ONU a través de la Unesco.

${ }^{6}$ Comité Internacional para la conservación y defensa del Patrimonio Industrial.
} 
uso de principios generales para facilitar e impulsar su documentación, protección y conservación. Así se establece que el Patrimonio Industrial incluye los sitios, complejos, estructuras, áreas y paisajes, la maquinaria utilizada, los objetos accesorios y los documentos que proveen evidencias y pruebas de la existencia de los diferentes procesos industriales, así como de las relaciones sociales generadas por éste en momentos históricos definidos (Engelking, 2014).
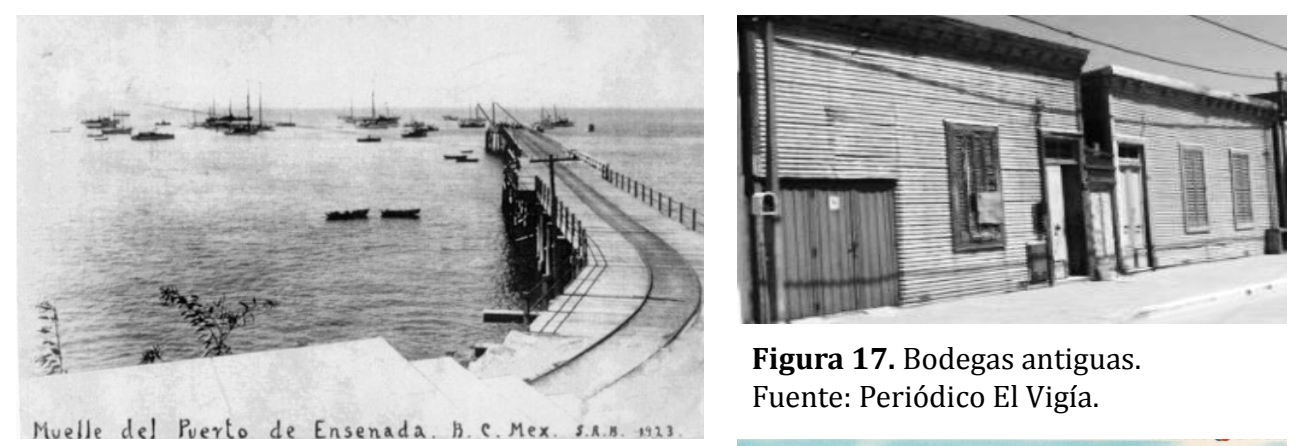

Figura 17. Bodegas antiguas. Fuente: Periódico El Vigía.

Figura 16. Muelle del puerto en 1923.

Fuente: Periódico El Vigía.
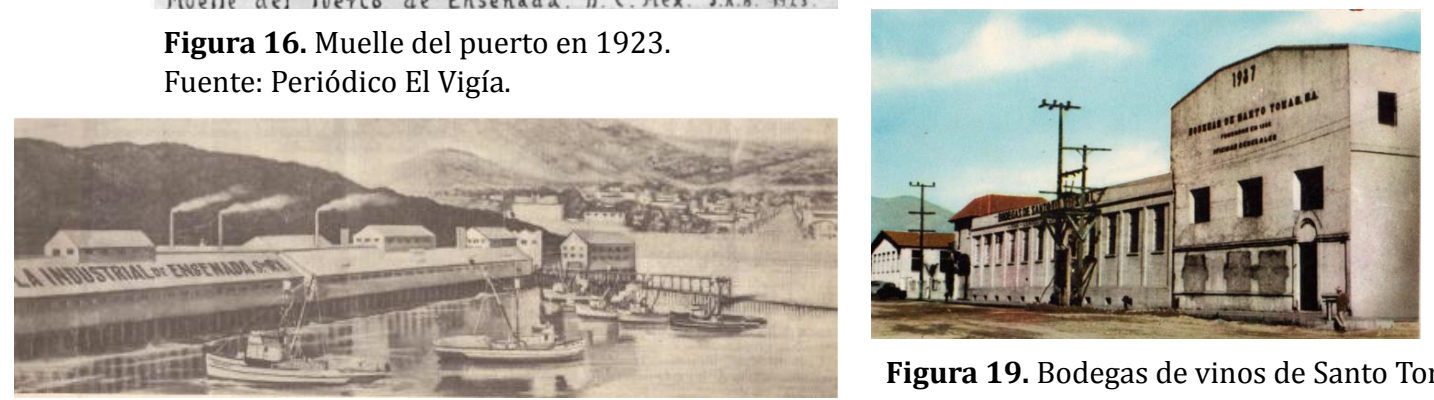

Figura 19. Bodegas de vinos de Santo Tomas.

Figura 18. Empacadora La industrial de Ensenada S. de R.L: Fuente: México en fotos

Fuente: ensenada.net

Respondiendo a la hipótesis inicial de que la traza urbana de Ensenada responde a un modelo urbanístico norteamericano concluimos que inclusive su lotificación y modulación inicial podría tener influencia en la forma en que la compañía desarrolló su proyecto, ya que el módulo seguirá siendo el mismo y los lotes responden a características similares solo presentan un ligero giro de la retícula respecto a su posición original. Referente a las edificaciones de la etapa industrial, reconocemos en una primera etapa un rico legado con ejemplos únicos de carácter patrimonial, creemos que son de enorme valor las iniciativas e investigaciones que han ido surgiendo en la Facultad de Arquitectura de la Universidad Autónoma de Baja California las cuales son los cimientos para valorar la cultura y buscar líneas de intervención generando oportunidades para su conservación.

El economista Miguel Ángel Álvarez Areces (2010), experto en patrimonio industrial menciona:

Los valores paisajísticos, las huellas industriales y las herencias artísticas se entremezclan en un espacio continuo. El Patrimonio Industrial ha adquirido un sentido que excede de lo estético o testimonial para convertirse en un núcleo de orden temporal y espacial frente al avance del olvido y la pérdida de la memoria del lugar. Los territorios inteligentes o "smartplaces" en los que viven comunidades activas son o han sido capaces de organizarse para inventar y alcanzar un consenso con respecto a un proyecto de futuro (Álvarez Areces 2010).

Habrá que reconocer las primeras muestras de identidad por parte de la ciudadanía e iniciativa privada en conservar las instalaciones industriales de las Bodegas de Santo Tomás. Hoy en día se ha declarado patrimonio cultural a un conjunto arquitectónico, en los términos de la ley de preservación del patrimonio cultural del estado de Baja California, en la categoría de zona protegida, modalidad de distrito urbano. El bien comprende 14 edificaciones de tipo industrial ubicados en la primera sección de Ensenada que remontan sus orígenes a principios del siglo XX, constituyendo un valor histórico, cultural, social, arquitectónico y artístico.

Las bodegas forman parte de la historia, costumbres y tradiciones de la ciudad, particularmente con relación a la producción del vino, la cual le brinda a Baja California un lugar privilegiado a nivel 
nacional en esta actividad. Las edificaciones están en la zona más antigua de Ensenada y forman parte de la traza original plasmada en el plano de 1886.

La conservación del patrimonio industrial es fundamental para el entendimiento de la historia de las poblaciones porque hace posible resaltar su identidad y ponerla al servicio de la propia comunidad, reconstruyendo edificios y diseñando itinerarios culturales para el beneficio urbano de toda ciudad.

\section{Consideraciones finales}

La ciudad de Ensenada representa una caso muy interesante de como se establece un proyecto urbano a través de una compañía extranjera en el territorio más alejado del centro de México. Sus cualidades urbanísticas como la racionalidad regular de su retícula y sus amplias avenidas que ordenan la ciudad responden al modelo norteamericano de colonización y poco tiene que ver con la tradición Novo hispana que se estableció en la mayoría del país, ya que en esta región se presentó una influencia que llego de norte a sur por medio del capitalismo extranjero.

Se reconoce que algunas edificaciones tradicionales se están demoliendo o están en deterioro, y que se está perdiendo parte de un patrimonio arquitectónico por la incompetencia de ciertas autoridades gubernamentales para manejar esta problemática. Existe un desconocimiento del valor que guardan las construcciones de Balloon Frame o cualquier otra construida en los inicios de la ciudad, una identidad reciente de la etapa industrial que se está borrando.

Es de vital importancia ampliar y difundir estudios sobre la historia reciente del urbanismo en el Noroeste de México, ya que se percibe cierto desconocimiento sobre el origen de este tipo de ciudades, las cuales poseen una identidad nueva pero no por ello menos valiosa en términos de apropiación del espacio y de las sociedades que conformaron, inclusive cuando éstas han sido diseñadas por compañías extranjeras. Es necesario nombrar y mostrar los proyectos modernos que consolidaron la frontera entre México y Estados Unidos, ya que esta región cuenta con una tradición industrial reconocida que deberá poco a poco ser valorada ya que a través de ella se estructuró y se modernizó este territorio. Por eso, los gobiernos locales y las políticas actuales de preservación deberán encarar el siglo XXI generando nuevos proyectos de valorización de trazados y edificaciones para poder así garantizar los valores y la identidad de esta parte de México.

Actualmente la ciudad de Ensenada cuenta con una población de aproximadamente 520.000 habitantes y su puerto turístico y comercial se enlaza principalmente con California, el Pacífico mexicano y con el continente asiático. Su región en los últimos años ha llamado la atención a nivel nacional por su belleza paisajística de viñedos en el Valle de Guadalupe, mostrando nuevas oportunidades de un territorio emergente rico en recursos.

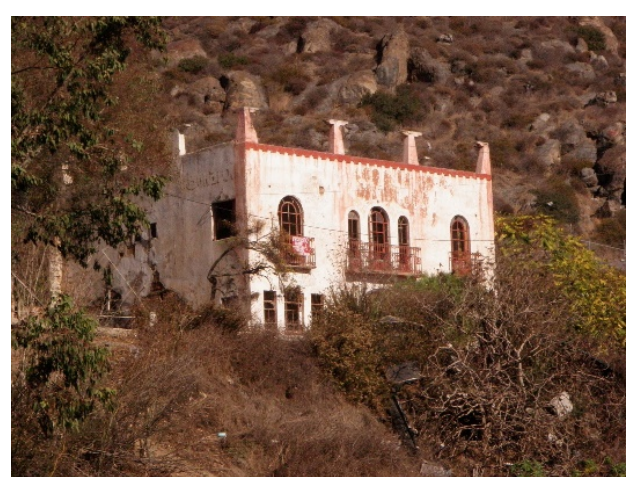

Figura 20. Edificio en ruinas en la entrada a la ciudad frente al puerto. Fuente: Fotografía de Luis Manuel Guzmán Muñoz 2013.

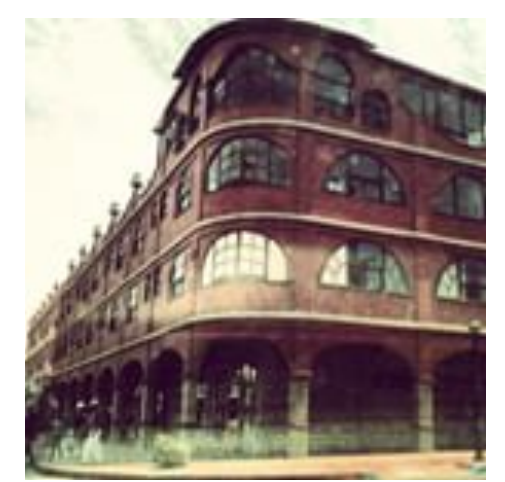

Figura 21. Antiguo edificio del Hotel Plaza. Fuente: Fotografía Enrique Gómez 2013.

Por tanto, se propone en una primera aproximación identificar elementos construidos en la etapa industrial que ayuden a narrar la historia urbana de la ciudad. Se espera también que este estudio sirva como una posibilidad de lectura de este territorio a través de la historia industrial, con la 
intención de dar un punto de vista diferente en la discusión sobre lo que pasa con estas ciudades modernas del Noroeste de México teniendo en cuenta las particularidades de cada una de ellas.

\section{Referencias}

Alarcón, E. (2000). “Estructura urbana de ciudades fronterizas". Nuevo Laredo- Laredo, Reynosa- Mc Allen, Matamoros- Brownsville. El Colegio de la Frontera Norte. pp 232. Tijuana [Baja California] México.

Álvarez Arececes, M. Á. (2010). “La herencia industrial y cultural en el paisaje: Patrimonio Industrial, Paisaje y Territorios Inteligentes". Revista Labor \& Engenho 4 (1) 78-100. [Campinas [SP] Brasil. ISSN:2176-8846].

Blasco, J. (2013). Claves de las ciudades norteamericanas (de colonización): cuadrícula, bidimensionalidad y "pecados originales". Urban Networks.

Burian, E. (2015). The Architecture and Cities of Northern Mexico from Independence to the Present. Austin: University of Texas Press.

Calderón, M., \& Geffroy, B. (2001). Un Siglo de Arquitectura en Ensenada (pp 1-87.). Ensenada [Baja California]: Fondo Editorial de Baja California.

Calderón, M. (2007). "Adecuación bioclimática de la vivienda tradicional de Ensenada, Baja California, México 1882-1930. PALAPA. Journal of Scientific Research on Architecture. Vol 2, No 1. 2007.

Debat, M. (2010). “Impresión y adaptación. La construcción de la identidad del área central de la ciudad de Córdoba”. Revista Identidades, Territorio, Proyecto y Patrimonio. No 2 pp 45-56. Barcelona España.

Engelking, S. (2014). "Una especie en extinción, la arquitectura industrial del siglo XX; la industria en la zona metropolitana de la ciudad de México y su entorno urbano". En Primer Coloquio Nacional: Los Procesos de Industrialización en México. INAH. 2014.

Heath, Hilarie (2011). "El malogrado proyecto del ferrocarril peninsular del Distrito Norte de la Baja California, 1887-1892". Estudios fronterizos. Versión impresa ISSN 0187-6961. vol.12 no.24 Mexicali jul./ dic. 2011

Jordán, Fernando (1956). El otro México, Biografía de Baja California. Tijuana [México]: Instituto de Investigaciones Históricas de Baja California, 2010.

Morris, A. E. J. (1994). History of Urban Form: Before the Industrial Revolution. Nueva York: Longman Technical and Scientific-John Wiley and Sons.

Padilla, A. (1998). Inicios Urbanos del Norte de Baja California, Influencias e ideas, 1821-1906. Mexicali Baja California: Universidad Autónoma de Baja California. 1998.

Piñera, D., \& Bejarano, A. (2011). “Expresiones arquitectónicas compartidas en la frontera de Baja California y California". Revista Culturales, vol.7, no14, Mexicali 2011.

Piñera, D. (1995). American and English Influence on the Early Development of Ensenada, Baja California México. San Diego [California] U.S.A.: Institute for Regional Studies of the Californias, San Diego State University.

Reps, John. W. (1979). Cities of the American West: A History of Frontier Urban Planning. John W. Reps. Princeton: Princeton University Press.

Ruiz, P. (2012). "Propuesta metodológica para analizar, conservar y reutilizar el patrimonio industrial”. Revista Boletín de Monumentos Históricos del Instituto Nacional de Antropología e Historia. Tercera época, num.25. México, D.F. 2011.

Romero, M. (1986). Noreste minero. La minería en Sonora, Baja California, Baja California Sur durante el Porfiriato. Plaza y Valdés Editores.

Sabaté, J. (2014). "Las medidas de los trazados ortogonales" Cuadernos de Investigación en Urbanismo. QRU. Trazados Urbanos. Grup de Recerca en Urbanisme. DUOT.UPC, Barcelona. 